\title{
Review
}

\section{My 50 years of research in particle physics}

\author{
By Hirotaka SuGAWARA ${ }^{* 1, * 2, * 3, \dagger}$
}

(Communicated by Toshimitsu YAMAZAKI, M.J.A.)

\begin{abstract}
Some of my work of the last 50 years in the field of theoretical particle physics is described with particular emphasis on the motivation, the process of investigation, relationship to the work of others, and its impact. My judgment is unavoidably subjective, although I do present the comments of other researchers as much as possible.
\end{abstract}

Keywords: Sugawara construction, Lee-Sugawara relation, supersymmetry CP violation, Kamiokande, population genetics

\section{Introduction}

Until now I have not tried to review what I achieved in the field of particle physics because obviously objective judgment can and should be done only by other researchers in the field. Nothing is harder than to try to be objective about yourself. However, I have been asked by the Japan Academy to do this rather awkward task, so I will present here a subjective description of my own work starting from the early 1960's to the present. I will try, as much as possible, to clarify what motivated me in each stage of my work and how it has been related to other people's work.

I want to emphasize the crucial role of informal, collaborative discussion among colleagues in the development of new ideas. Ever since my graduate student days I have been stimulated and challenged by the free exchange of ideas with my colleagues near and far. I think these free and open exchanges are the foundation of discovery. Many people contribute to the development of each new idea. I would like to give some examples. I will quote some comments by other people to avoid excessive subjectivity, but I doubt if I can be even subjectively successful in the

\footnotetext{
*1 JSPS Washington Office, Washington, DC, USA.

*2 Johns Hopkins University, Maryland, USA.

*3 KEK, High Energy Accelerator Research Organization, Ibaraki, Japan.

$\dagger$ Correspondence should be addressed: H. Sugawara, JSPS Washington Office, 2001 L Street NW \#1050, Washington, DC 20036, USA (e-mail: sugawara@jspsusa.org).
}

attempt. I would, therefore, rather concentrate on the motivation for each work and the way the work was accomplished. I would be more than happy if this paper were useful to young researchers in pursuing their own research activities.

I will first describe some papers done during my $\mathrm{PhD}$ student days at the University of Tokyo, then as a postdoctoral fellow in the United States and Europe. After that I discuss my work in the 1980's and 90's, followed by my current interests.

In the early 1960's theorists became very skeptical of field theory, especially with regard to understanding strong interactions. Dispersion theory was seriously considered as a candidate to replace the entire field theory scheme; Geoffrey Chew of University of California at Berkeley was a prime promoter of this direction. ${ }^{1)}$ In retrospect I would say that the efforts of S-matrix theorists eventually gave birth to string theory, due mostly to the discovery of duality at the phenomenological level, ${ }^{2)}$ a subsequent paper by G. Veneziano ${ }^{3)}$ and Nambu, and Goto's imaginative approach to its interpretation.4),5) (Superstring theory gained momentum at a much later time when the cancellation of anomaly was discovered by M. B. Green and J. H. Shwartz. ${ }^{6}$ )

There was another direction people were pursuing during this period; symmetry property of particle interactions stemming partly from the problem of particle classifications ${ }^{7-10)}$ and partly from the related problem of understanding the property of weak interactions. ${ }^{11-13)}$ As a graduate student I was supposed to understand both of these activities and I wrote several papers on these two approaches. I will 
select one paper from the dispersion approach and three papers from the symmetry approaches.

Although the perturbative approach to strong interactions was widely disputed during this period, there was also some doubt about the idea of totally abandoning field theory. Therefore, we tried to find some way, avoiding the perturbative calculations, to see if field theory still could be useful in understanding strong interactions.

I was very much involved in these kinds of activities throughout my entire post-doctoral days in the United States and Europe. I will choose two papers from this period and describe them in more detail.

I might say that the 1970's was the period of complete resurgence of field theory through the discovery of $\mathrm{QCD}^{14)-17)}$ and the Standard Model of weak and electromagnetic interactions. ${ }^{18)-20)}$ On the other hand, S-matrix theory was completely transformed and, combined with supersymmentry idea and gravity theory, became superstring theory, although its validity still remains to be seen. Through the entire period of 1970's and 1980's I was interested in the phenomenological aspect of the Standard Model, especially in its symmetry structure. Its extension to the grand unification scheme ${ }^{21)}$ was particularly attractive in view of its possibility for further generalization towards superstring theory although, as every researcher in the field should admit, we are still far from its understanding. I will pick two papers from this category. On the other hand, I did not lose my interest in superstring theory; what I had done at earlier stage did have something to do with it, although that was not my intention when I worked on it.

From 1990 to the present time I became very much involved in managing KEK, then Sokendai and now the JSPS Washington DC office, so I have had much less time for my own scientific activities. Nevertheless, somehow I succeeded in maintaining a strong interest in the research and have even broadened my scientific viewpoint by being exposed to many different, but related fields such as population genetics, environmental studies, brain research etc. I will conclude by describing some works in which I am still engaged.

\section{As a PhD student}

My supervisor, Professor Hironari Miyazawa, was one of the most prominent S-matrix theorists and we students were under his strong influence. An important task of S-matrix theorists around that time was to explain the hadronic cross sections due to strong interactions, especially energy dependence and angular distribution. Experimentally, the total cross section was found to be more or less independent of energy for most of the channels and the concept of Pomeranchuk Regge pole ${ }^{22)}$ was introduced to explain this phenomenon. Generically the Regge pole (singularity in the plane of analytically extended angular momentum ${ }^{23)}$ ) position depends on the energy of the channel under consideration. In the description of high energy scattering, the Regge pole in the crossed channel is important and this gives the angular dependence of the scattering amplitude. What was found experimentally was that the elastic scattering angular distribution was energy independent, contrary to the hypothesis of moving Regge pole. So the question was: "Is it possible that the pole position of the Pomeranchuk pole is energy independent?" I tried to prove this possibility.24)

For S-matrix theorists the only physical or mathematical tools allowed were the analyticity and the unitarity of the scattering amplitudes. What I could prove in this paper was that if the pole position as a function of energy has a zero slope at zero energy, then all the higher order derivatives will also disappear. In retrospect, through this work I personally learned the power of analyticity when combined with the unitarity, but the impact of this paper must have been rather limited.

Throughout the 1960's we saw a rather dramatic increase in the number of "elementary particles" or resonances, and it was clear that we should abandon the idea of all these being "elementary". The more practical, but related question was to classify all these particles and we knew some group theory must be useful, based on the experience in other fields such as crystallography. A concrete idea came from the Nagoya Group, ${ }^{8)}$ Gell-Mann, and Ne'eman.9),10) I got interested in the question of what role group theory might play in understanding weak interactions because we knew the weak interactions do not respect these symmetries. I decided to study the non-leptonic weak decays of hyperons as an example because these processes were least understood and I wanted in a way to avoid going into the detailed current-current structure of the weak interactions. The idea was very simple: We already knew that the mass term which breaks the $\mathrm{SU}(3)$ symmetry of Nagoya-Gell-Mann-Ne'eman is dominated by 
the octet representation of the group.9),25) I assumed that the same thing would happen to the weak Hamiltonian and then obtained a certain sum rule which turned out to rather nicely fit the experimental data. ${ }^{26)}$ I learned from Y. Hara who was staying at Caltech at that time that M. Gell-Mann had gotten interested in my work and had obtained the same sum rule, at least for the s-wave amplitude without assuming the R-invariance. ${ }^{27}$ ) What was surprising to me was that B. Lee who was then at the University of Pennsylvania had obtained exactly the same sum rule ${ }^{28)}$ with exactly the same assumptions. I learned two lessons from this work. First was the joy of explaining experimental data and the second was the fact that people can come up with the same idea in physics quite independently of each other. These two lessons stayed in me throughout my entire research career.

I got more and more impatient just working within the framework of Nagoya - Caltech SU(3). For me, at least at that time, group theory was just a convenient tool to classify particles. I was amazed by the similarity of classifying bosons and fermions using the same group $\mathrm{SU}(3)$. I thought there must be some dynamical reason and consequently there must be symmetry of those interactions which govern the dynamics which in turn leads to the bosonfermion similarity. I was certainly aware of the fact that the Poincare symmetry must be included and the difference of boson-fermion statistics should also be overcome. After some consideration, I talked to Professor Miyazawa about my idea. Although he was the outstanding S-matrix theorist, he was very open-minded to all kinds of physics ideas and he showed interest in what I was proposing. We decided to write a joint paper on the subject and we eventually wrote two. ${ }^{29)}$

In retrospect, I should say that our work differed from the current version of supersymmetry in two points; As the equation (7) of the second paper shows, our idea was to create a situation in which we can have either commutation or anti-commutation relation. Our way of thinking was that symmetry should be experimentally valid only when the statistics does not matter. Therefore, we should be able to choose either of them. The second difference is that we tried to incorporate Lorentz symmetry, rather than translation symmetry into our supersymmetry. Nevertheless, I believe we were on a right track towards the current supersymmetry. In fact, at a later stage, Professor Miyazawa pushed forward this idea by himself and became the inventor of supersymmetry. ${ }^{30), 31)}$ I hope that our work played at least a precursor role to the idea of supersymmetry.

I quote from a short note by Miyazawa entitled, "Birth of Superalgebra"

(http://www7.ocn.ne.jp/ miyazaw1/papers/susy4.pdf):

In nineteen sixties the top topic was the group theory and the classification of elementary particles. Bunji Sakita, the inventor of the $S U(6)$ theory, visited us at the University of Tokyo, and excited our interest in this theory.

The SU(6) theory combines particles with spin 0 and 1, or spin 1/2 and 3/2 in one representation. I noticed a beautiful parallelism between bose particles and fermi particles and wondered if they could be combined in one representation. Ignoring statistics, this is easy to do. All existing elementary particles can be expressed by the adjoint representation of the SU(9) group. Hirotaka Sugawara and I wrote a short note entitled "SU(9) Symmetry" published in $1965[1]$.

Of course, I was not satisfied with this scheme since this only works for one particle states, i.e., in the case of Boltzmann statistics. I looked for a real mathematical scheme and soon found that a hamiltonian of the form

$$
H=m\left(X b^{\dagger} b+X f^{\dagger} f\right)
$$

where $b$ stands for boson annihilator and f fermion annihilator, has usual conserved quantities consisting of $b^{\dagger} b$ and $f^{\dagger} f$. In addition $H$ also commutes with spinor quantities of the form $b^{\dagger} f$ and $f^{\dagger} b$. The set of all conserved quantities of these forms closes on commutators and anticommutators. Thus I arrived at a new algebraic scheme with commutators and anticommutators. This paper was published in Progress of Theoretical Physics[2].

Since its publication I received some suggestions that by introducing anticommuting quantities the algebra can be reduced to an ordinary Lie algebra. In such a scheme, one can construct only one particle state and no more. This is the case of Boltzmann statistics and I paid no attention to them.

In 1968 I was at the University of Chicago, generalized the algebra to include the $S U(6)$ and introduced an algebra which I called $V(6,21)$. Here $V$ meant beyond Unitary. This would now be called SU(6/21). I sent it to Physical Review[3]. The referee commented that this paper was very original and should be published in one form or another even though the result was not terribly interesting, and added that $I$ should start from a simpler example. Actually this was already done in my previous paper, so I stuck to the complicated model. 
While writing this paper I did not know how to call the algebra of this type. One day I called a professor of mathematics, Ichiro Satake, explained my algebra and asked him if such commutater-anticommutater algebra exists in the mathematical community. He replied that an algebra with anticommutators only was often called as Jordan algebra but he had never seen such a mixture. He was not interested in this algebra.

About the same time I also explained this to Murray Gell-Mann who remarked: what is the all types of such algebra? The compact Lie groups are limited to a few cases, the orthogonal groups, unitary groups, symplectic groups and some exceptional ones. Similarly, all types of the mixture algebra could be listed up. I regarded this an interesting mathematical problem.

However, I wanted to try a more physical project, i.e., to formulate the scheme in a relativistically invariant way. I tried first to write down an example of a relativistically invariant lagrangean that accepts boson-fermion symmetry. This was not easy, and before reaching the goal, I lost interest in this project. I thought that such relativistic boson-fermion symmetry (now called supersymmetry) could be formulated mathematically but it would not be the fundamental symmetry of physics. If it were, the fundamental particles must consist of fermions and bosons. This contradicts the principle that the fundamental objects must be very few.

[1] H. Miyazawa, H. Sugawara, Prog. Theor. Phys. $33(1965)$ rr71.

[2] H. Miyazawa, Prog. Theor. Phys. 36(1966)1266.

[3] H. Miyazawa, Phys. Rev. 170(1968)1586.

\section{As a post doctoral fellow}

In 1965 I became a postdoctoral fellow at Cornell University. A few months before I took the job, Adler $^{32)}$ and Weissburger ${ }^{33)}$ independently published a very interesting work which showed that the framework of local field theory can be valid and even useful in understanding strong interactions. The basic idea is to use various local currents which can lead to the symmetry of interactions as a tool to study strong interaction. This was so interesting that I started to use a slightly different technique regarding the current algebra and applied this to the process of non-leptonic hyperon decays; this was a process which I was familiar with since my graduate student work. I was able to reproduce some sum rules, ${ }^{34)}$ including the one which I had derived when I was a student. All the sum rules were experimentally verified and so I was convinced, together with many other theorists, that at least the basic framework of field theory must be correct. What surprised me again here was that M. Suzuki, my classmate from the University of Tokyo, obtained the same result ${ }^{35)}$ using the same technique, although he was then at Caltech and I have no memory of us talking about this subject. We must have shared some philosophical attitude towards physics as students of Miyazawa.

M. Gell-Mann ${ }^{36)}$ and G. Zweig ${ }^{37)}$ introduced the idea of the quark in 1963 as a purely mathematical object to explain the dynamics and symmetry of particle interactions. The successful use of current algebra in describing particle interactions led many researchers to the idea of using only currents, but not the quark field, as the fundamental objects of particle theory. This idea looked quite adequate for quark theory since the quarks do not exist as a particle but as constituent of currents. At first, I was not clear what the framework should be. When C. Sommerfield of Yale University gave a talk at Berkeley and argued that the right way is to write the energy momentum tensor in terms of currents, I understood that it is the way to replace the conventional action theory. Sommerfield did not show the form of the energy momentum tensor in terms of currents in a consistent way, so I started to work on this issue. The first and foremost difficulty was to treat the so-called Schwinger term in current algebra. The simple Lagrangean model for quarks inevitably gave a diverging operator form for it. It was, therefore, useless if one wants to start from the explicit current algebra. After all kinds of trial and error, I finally succeeded in writing the energy-momentum tensor in terms of currents when we give up the q-number Schwinger term. ${ }^{38)}$ I was rather skeptical about whether I had succeeded in writing a quark theory without using the quark field, because of this cnumber Schwinger term. But, I decided to publish the result. The reaction was more than I expected. I want to quote a statement from D. J. Gross's Nobel Lecture in 2004:

In the spring of 1968 Curtis Callan and I proposed a sum rule to test the then popular "Sugawara model," a dynamical model of local currents, in which the energy momentum tensor was expressed as a product of currents. The hope was that the algebraic properties of the currents and the expression for the Hamiltonian in terms of these would be enough to have a complete theory. Our goal was slightly more modestto test the hypothesis by exploiting the fact that in this theory the operator product expansion of the currents contained the energy momentum tensor with a known 
coefficient. Thus we could derive a sum rule for the structure functions that could be measured in deepinelastic electron-proton scattering [18].

Eventually, however, the current-current Hamiltonian approach was replaced by more traditional Lagrangean formulation-QCD to which Y. Nambu, ${ }^{14)}$ G. t'Hooft and M. Veltman, ${ }^{15)}$ D. J. Gross and F. Wilczek, ${ }^{16)}$ H. D. Politzer, ${ }^{17)}$ and many other people had contributed. The quark was supposed to be confined dynamically, rather than just being a mathematical object.

The current-current Hamiltonian approach turned out to have a rather unexpected, but very interesting application in string theory. I quote the following statement by M. Gell-Mann from his closing talk at the Second Nobel Symposium on Particle Physics, June 1986:

Around the same time, 1966, after working for five years or so on current algebra, I suggested repeatedly that it would be wonderful if we could express the whole dynamics by means of current algebra, adjoining the energy density to the algebra of the internal charge and current densities, with the energy density expressed in terms of the charge and current densities, particularly in a light-cone frame.

In 1968, in a bar in Ankara, I met Sugawara, who told me he had created such a model. I was delighted, but over time all of us became discouraged when we learned that it had really nice properties only in two dimensions. Today, as David Olive described so eloquently, such two-dimensional systems have turned out to be fundamental. And, the expression "twodimensional" now rouses great hope where once it led to disappointment.

I should say that the current-current construction of the energy-momentum, which is usually called the Sugawara construction, should, in fact, be called Gell-Mann-Sommerfield-Sugawara construction, as all these people contributed to it in their own way.

\section{Standard model}

Throughout the 1970's and the 1980's we saw the resurgence of local field theory with a particular emphasis on the importance of the gauge principle. Most of the particle interactions became understandable within the framework of gauge theories. A surprisingly simple model of the weak and electromagnetic interactions by S. Weinberg ${ }^{18)}$ and A. Salam ${ }^{19)}$ turned out to be correct, as the discovery of neutral currents $^{39)}$ dramatically demonstrated.

From the beginning my own interest was in studying the defects of the Standard Model: the
Higgs interaction was not understood as gauge interaction and its origin is left untouched in the Standard Model. As a related problem, the question of the flavor degrees of freedom still remains.

It was well known that CP symmetry cannot be violated in gauge interactions and that the Higgs interaction was the only way to violate CP symmetry within the Standard Model. M. Kobayashi and T. Maskawa investigated this issue and proposed several versions to solve this problem.40) S. Pakvasa and I pursued their three quark version and studied its physical consequences. ${ }^{41)}$ I quote from S. Pakvasa' note in the special issue of Progress of Theoretical Physics Commemorating the Nobel Prize awarded to M. Kobayashi and T. Maskawa:42)

In 1972, Kobayashi and Maskawa ${ }^{10)}$ turned their attention to the question of $C P$ violation and how to incorporate it in a renormalizable theory of weak interactions, specifically the $S U(2) \times U(1)$ model due to Glashow, Salam and Weinberg. ${ }^{11)}$ Although they did not cite the GIM (Glashow-Iliopoulos-Maiani) paper, ${ }^{12)}$ the contents of it were surely known to them, including the absence of flavor changing neutral currents (FCNC). In their paper, they started by observing the fact that in this scheme with two families, there is no room for a non-trivial phase that could give rise to observable CP violation. The weak charged current for quarks is given by

$$
J_{\mu}=(\bar{d} \bar{s})_{L} U_{\gamma_{\mu}}\left(\begin{array}{l}
u \\
c
\end{array}\right)_{L}
$$

In Eq. (1), $U$ is a unitary $2 \times 2$ matrix which in general has three phases and one angle. But the three phases can be absorbed into the definition of three quark fields, and only the one mixing angle, the Cabibbo angle remains. ${ }^{13)}$ They then ask how can one introduce a non-trivial phase, giving rise to $C P$ non-conservation with minimum change in the model. They go on to try various options.

One is to introduce a right handed doublet of quarks (and leptons to preserve anomaly cancellation). In this case, there is indeed room for an extra phase which cannot be transformed away and can give rise to the observed $C P$ violation in $K$ decay. They observed that there are some phenomenological problems with such a scenario. This possibility was raised and pursued already slightly earlier by Mohapatra, ${ }^{14}$ ) who also found that there needs to be further proliferation of fields in such a model. They also briefly considered other models with right handed currents and dismissed them as being not satisfactory.

The second possibility is to increase the number of scalar fields beyond the minimal single Higgs doublet by one, and have two scalar fields. In this case also 
there is an extra phase which can give rise to CP violation. A few months earlier, Lee ${ }^{15)}$ had considered such a model in connection with a theory of spontaneous $T$ violation.

Finally, in the last page of the paper, they raise the third possibility of having three families of quarks and leptons. In this case, they observe that the $3 \times 3$ unitary matrix describing the mixing in the charged current coupling has 6 phases (and three rotation angles), but only five of the phases can be absorbed in the definition of the quark fields, thus leaving one nontrivial phase which could give rise to observable CP violation. They give an explicit parameterisation of the matrix.

In summer 1975, when Hirotaka Sugawara arrived in Hawaii for his annual two month visit, he suggested that we work on CP violation, and specifically study the proposal in the K-M paper. We found that it was indeed possible to obtain a correct value for the $K_{L}$ decay rate with a reasonable choice of the new Cabibbo-like angles and the phase in the K-M matrix. It was also possible to estimate the $\varepsilon^{\prime}$, albeit crudely, and show it was small, thus leading to an approximately "superweak" model (at least in the K-system) of $C P$ violation. Thus we showed that the $K-M$ proposal was indeed a viable description of the known $C P$ violation at the time. We submitted the paper (to Physical Review Letters) in September 1975. It was eventually accepted for Physical Review and appeared in print in July 1976. ${ }^{22)}$ Ours was only the third paper to cite the K-M paper.

A paper by Maiani appeared shortly after ours in which he independently reached the same conclusions we did. ${ }^{23)}$ In a few months, a long detailed paper by Ellis, Gaillard and Nanopoulos came out. ${ }^{24)}$ This contained very detailed, careful calculations of $C P$ violating effects in $K$ decay, and also considered possible $C P$ violation in other heavy quark systems. All three papers agreed about the fact that $C P$ violation in $K$ decay can be accounted for, and that the ratio $\varepsilon^{\prime} / \varepsilon$ is very small, and hence that predictions are approximately "superweak". None of the papers found any large effects with which the model could be easily and quickly checked.

The K-M paper had now been "discovered" and began to be cited widely, and became part of the standard lore along with the papers of Glashow, Salam, Weinberg, and GIM!

The family or the flavor problem of quarks and leptons is closely related to the symmetry of the Higgs interactions. It does not seem to be related to gauge symmetry, at least in the low energy region. In such a situation it is natural to consider some kind of discrete symmetry to avoid the appearance of Nambu-Goldstone bosons. S. Pakvasa and I initi- ated such an approach to the family problem and we had some limited success. ${ }^{43)}$ This approach has been revived recently, especially in the lepton sector.

Non-gauge symmetry is destined to be broken at higher energies and the outstanding problem is to find the mechanism of this symmetry breaking. Until then we will not be able to understand the family problem, even if discrete symmetry is the right way to proceed. Supersymmetry has been introduced into the framework of the standard model to explain the hierarchy problem, and the supersymmetric standard model has become a standard platform for phenomenologists to study. The outstanding issue since early 1990's is not a theoretical one, but the experimental verification of the standard model: the discovery of the Higgs particle and the superparticles. For almost twenty years I was involved in encouraging those experimental projects in various ways, first in managing the KEK physics division and then as director general of KEK, now known as the High Energy Accelerator Research Organization.

B-Factory, J-PARC and Kamioka. In an article in a special issue of Progress of Theoretical Physics I described the process by which the KEK B-Factory was started. ${ }^{44)}$ Another project I started as KEK director is the current J-PARC. I hope to be able to talk about that process another time.

A related interest of mine was in Grand Unified Theory. I gave a summary talk on this issue at the High Energy Physics Conference which was held in Madison, Wisconsin in 1980. ${ }^{45}$ ) When M. Yoshimura came up with the idea ${ }^{46}$ in 1978 that the baryon number of the universe can be explained within the framework of Grand Unified Theory, it was clear to me and to many people in the field that the crucial parameter for Grand Unified Theory is the rate of proton decay. I asked M. Koshiba to design such an experiment and he and his group came up with a reasonable project. ${ }^{47)}$ I also asked T. Nishikawa, then the KEK director, to help us fund this project. He was more than enthusiastic, and, thanks largely to his efforts, the project was funded. The Kamioka facility was taken care of by KEK under the leadership of K. Takahashi and the detector belonged to the University of Tokyo. Koshiba's group concentrated on proton decay and the neutrino physics was done by researchers of Osaka City University, at least in the beginning.

Initially, J. Arafune and myself were among the members of the Kamiokande Group and our names 
were on the author list. Later we learned that our names had been deleted from both lists. However, Arafune and I have never lost our interest in the physics of Kamiokande. In fact, much later Arafune succeeded in funding the Superkamiokande project when he was the Director General of the Institute of Cosmic Ray Research and, as Director General of KEK I too strongly supported various Kamiokande projects.

\section{Current interests}

Common feature of superstring theory, climate change models and the brain models is that they lack sufficient data to support the various models being proposed. I am trying to address that problem by inventing new ways of collecting more data and by trying to find missing links that connect the models to the data in each of these area. A model becomes a true theory only after being tested a sufficient number of times by the data and after showing the power of predicting the output data from a known set of inputs.

The S-matrix approach of the 1960's was turned into superstring theory with the discovery of anomaly cancellations $^{6}$ ) and the superman-like performance of E. Witten. Its relevance to the unification of all interactions, including gravity, has become widely recognized. It became a fascinating field of mathematical physics, but, unfortunately, without much relevance to the real world, at least for now. It is getting clearer and clearer that superstring theory includes non-string extended objects as fundamental as string itself. We also know that the vacuum state of string theory is intractably degenerate, although some of them seem to lead to an object which resembles our real world.

To my mind, the string formulation does not seem to be adequate for describing this situation, just as the idea that protons and neutrons are elementary particles lost its foundation in the 1960's when many resonances were found, although the finding in this case was in experiment, rather than in theory.

Is there any alternative then? I would say yes. There were proposals in 1997 by two independent groups; both are called matrix formulations of superstring theory. One is by T. Banks, W. Fischler, S.H. Shenker and L. Susskind ${ }^{48)}$ and the other is by N. Ishibashi, H. Kawai, Y. Kitazawa and A. Tsuchiya. ${ }^{49)}$ The second is closely related to the Eguchi-Kawai's reduced Yang-Mills theory. ${ }^{50)}$ Superstring theory is defined as some kind of limit of their matrix models.

I interpret the matrix model to be the theory of space-time with non-commuting coordinates. ${ }^{51)}$ It turns out that space-time can be naturally considered to be composed of integers or at most of rational numbers, rather than being a continuum. The reduced Yang-Mills equation is interpreted as an elliptic curve, thus implying an exciting possibility of relationship between physical theory and number theory in the most fundamental manner. There is also a convenient way to define gravity and gauge fields within this scheme as an excitation of spacetime. The vacuum state corresponds uniquely to the state of no matter and no space-time. The question is how we can deduce a low energy theory, starting from this formulation. I do not have the solution yet, but I believe this is the right way to proceed in the future.

Biology, population genetics, brain research, and nuclear disarmament. My contact with biologists when I was working in Sokendai as one of the Executive Directors made me very much interested in biology. The physical or chemical property of DNA in connection with the role of electrons in information transfer was one of the subjects Ikemura suggested that we work on together. Our preliminary research is in a report on quantum physics. ${ }^{52)}$ Another subject I am involved in is population genetics. As is well known, the role of mating, which, of course, must be fundamental in genetics, was carefully avoided in the mathematical formulation of population genetics initiated by such people as S. Wright, J.B.S. Haldane, R.A. Fisher and M. Kimura because of its complexity.

Takahata and I formulated a way to incorporate mating into the theory. ${ }^{53)} \mathrm{My}$ current interest is to extend this formulation to epigenetic processes.

Brain research is attracting many physicists, including myself. It is a fascinating subject but, unfortunately, we still have to depend on very poor data samples in this field. As far as I can see, data taking is most important before creating any kind of brain models. PET or MRI imaging can reach the space resolution of millimeter range but the size of the neurons is of the order of microns. Fluorescence can do better, but cannot reach the depth of millimeter order beyond the surface. I am suggesting the use of a radioactive material like ${ }^{26} \mathrm{Na}$ which could replace PET when some obstacles are overcome. 
With respect to data taking, environmental research is another field where lack of data is hampering the advancement of the field. It is my personal view that excessive dependence on so-called climate change models is rather unhealthy because we need data, especially that of the upper layer atmosphere. It is desirable to establish some sort of constant observational device to monitor the infrared absorption in a wide range of wave lengths and also to measure the amount of albedo. I am making my own proposal and giving talks in some US national laboratories about this.

Lastly, it is probably one of the duties of older physicists like me to be seriously involved in the issue of nuclear disarmament. I have completed some technical studies on the issue, but by far the most important thing about nuclear weapons is to understand the humans who fabricate and use such deadly weapons. I am, therefore, working with chemists, biologists, historians, anthropologists, ethnologists, political scientists, philosophers, politicians and bureaucrats to understand whether there is a way to eliminate such horrible weapons and make our world a safer place to live. ${ }^{54)}$

\section{Acknowledgment}

As I described in my essay, informal collaborative discussions have been at the center of my work in physics within Japan and around the world. In addition to the people I have already mentioned I have very much enjoyed working with many other people.

When we were at Cornell University under the strong influence of H. Bethe, F. von Hippel and I wrote a paper on the nuclear potential. I worked with T. Eguchi and wrote a rather well appreciated paper on Nambu's theory of spontaneous symmetry breaking. I am particularly grateful to Professor Nambu for inviting me to Chicago various times and patiently listening to my mostly crazy ideas. I also enjoyed working with J. Arafune tremendously in the Institute of Nuclear Studies, University of Tokyo and also at KEK. My collaboration with Y. Shimizu went beyond physics and we are still working together on the issue of "War and Peace". I spent a memorable time in Europe working with such people as P. Ramond, J. Nuyts, E. Cremmer etc. I also enjoyed working with such young people (meaning younger than myself), such as T. Kaneko, H. Hagura etc.

Many people helped me in managing KEK. Especially, I could not have managed KEK without the help of three deputy directors K. Kikuchi, K. Takahashi and Y. Kimura, each in a different time. S. Iwata managed the physics division almost entirely through my directorship. The wise advice of $\mathrm{K}$. Higashijima, Y. Kamiya, M. Kihara, M. Kobayashi, K. Nakamura, F. Takasaki, N. Toge, S. Wakatsuki, S. Yamada, M. Yoshioka and many, many others are all greatly appreciated. I became able to observe high energy physics community rather objectively thanks greatly to S. Traweek.

My last appreciation goes to Professor Miyazawa who taught us physics and how we should participate in creating new physics and also to Professor Nishikawa who taught me how to manage such a big laboratory as KEK The late Professor Yamanouchi once told me that Miyazawa and Nishikawa were the two most outstanding students of the University of Tokyo Physics Department throughout its entire history until his time. I am the lucky person whose professional career was more or less determined by the influence of those two most outstanding human beings.

\section{References}

1) Chew, G.F. (1961) S Matrix of Strong Interactions: Lecture Notes and Reprint Volume. Benjamin, New York

2) Dolen, R., Horn, D. and Schmid, C. (1968) Finiteenergy sum rules and their application to $\pi N$ charge exchange. Phys. Rev. 166, 1768-1781.

3) Veneziano, G. (1968) Construction of a crossing-symmetric, Regge-behaved amplitude for linearly rising trajectories. Nuovo Cimento A 57, 190-197.

4) Nambu, Y. (1970) In Proc. Intern. Conf. on Symmetries and Quark Models (ed. Chand, R.). Gordon and Breach, New York, p. 269.

5) Goto, T. (1971) Relativistic quantum mechanics of one-dimensional mechanical continuum and subsidiary condition of dual resonance model. Prog. Theor. Phys. 46, 1560-1569.

6) Green, M.B. and Schwarz, J.H. (1984) Anomaly cancellations in supersymmetric $D=10$ gauge theory and superstring theory. Phys. Lett. 149B, $117-122$.

7) Sakata, S. (1956) On a composite model for the new particles. Prog. Theor. Phys. 16, 686-688.

8) Ikeda, M., Ogawa, S. and Ohnuki, Y. (1960) A possible symmetry in Sakata's model for bosons-baryons system. II. Prog. Theor. Phys. 23, 1073-1099.

9) Gell-Mann, M. (1961) Symmetries of baryons and mesons. Phys. Rev. 125, 1067-1084.

10) Ne'eman, Y. (1961) Derivation of strong interactions from a gauge invariance. Nucl. Phys. 26, 222-229.

11) Maki, Z., Nakagawa, M. and Sakata, S. (1962) Remarks on the unified model of elementary particles. Prog. Theor. Phys. 28, 870-880.

12) Gell-Mann, M. and Levy, M. (1960) The axial vector current in beta decay. Nuovo Cimento 16, 705-726. 
13) Cabibbo, N. (1963) Unitary symmetry and leptonic decays. Phys. Rev. Lett. 10, 531-533.

14) Han, M.Y. and Nambu, Y. (1965) Three-triplet model with double $S U(3)$ symmetry. Phys. Rev. 139B, 1006-1010.

15) t'Hooft, G. and Veltman, M. (1972) Regularization and renormalization of gauge fields. Nucl. Phys. B 44, 189-213.

16) Gross, D.J. and Wilczek, F. (1973) Ultraviolet behavior of non-Abelian gauge theories. Phys. Rev. Lett. 30, 1343-1346.

17) Politzer, H.D. (1973) Reliable perturbative results for strong interactions? Phys. Rev. Lett. 30, 13461349 .

18) Weinberg, S. (1967) A model of leptons. Phys. Rev. Lett. 19, 1264-1266.

19) Salam, A. (1968) In Proceedings of the Eighth Nobel Symposium on Elementary Particle Theory (ed. Svartholm, N.). Almquvist and Wiksell, Stockholm, p. 367.

20) Glashow, S.L. (1961) Partial-symmetries of weak interactions. Nucl. Phys. 22, 579-588.

21) Georgi, H. and Glashow, S.L. (1974) Unity of all elementary-particle forces. Phys. Rev. Lett. 32, $438-441$.

22) Okun, L.B. and Pomeranchuk, I. (1956) Zh. Eksperm. I Teor. Fiz. 30, 424 [English translation, (1958) Soviet Phys. JETP, 7, 499].

23) Regge, T. (1959) Introduction to complex orbital momenta. Nuovo Cimento 14, 951-976.

24) Sugawara, H. (1963) Non-shrinking diffraction scattering and the Nambu-Sugawara-Miyazawa hypothesis. Prog. Theor. Phys. 30, 404-406.

25) Okubo, S. (1962) Note on unitary symmetry in strong interactions. Prog. Theor. Phys. 27, 949-966.

26) Sugawara, H. (1964) A new triangle relation for nonleptonic hyperon decay amplitudes as a consequence of the octet spurion and the $R$ symmetry. Prog. Theor. Phys. 31, 213-221.

27) Gell-Mann, M. (1964) Nonleptonic weak decays and the eightfold way. Phys. Rev. Lett. 12, 155-156.

28) Lee, B.W. (1964) Transformation properties of nonleptonic weak interactions. Phys. Rev. Lett. 12, 83-86.

29) Miyazawa, H. and Sugawara, H. (1965) Symmetry SU(9). Prog. Theor. Phys. 33, 771-772; Miyazawa, H. and Sugawara, H. (1965) Intrinsically broken $\tilde{U}(18)$ symmetry. Prog. Theor. Phys. 34, 263-273.

30) Miyazawa, H. (1966) Baryon number changing currents. Prog. Theor. Phys. 36, 1266-1276.

31) Miyazawa, H. (1968) Spinor currents and symmetries of baryons and mesons. Phys. Rev. 170, 15861590.

32) Adler, S.L. (1965) Calculation of the axial-vector coupling constant renormalization in $\beta$ decay. Phys. Rev. Lett. 14, 1051-1055.

33) Weissburger, W.I. (1965) Renormalization of the weak axial-vector coupling constant. Phys. Rev. Lett. 14, 1047-1051.

34) Sugawara, H. (1965) Application of current commutation rules to nonleptonic decay of hyperons. Phys. Rev. Lett. 15, 870-873.
35) Suzuki, M. (1965) Consequences of current commutation relations in the nonleptonic hyperon decays. Phys. Rev. Lett. 15, 986-989.

36) Gell-Mann, M. (1964) A schematic model of baryons and mesons. Phys. Lett. 8, 214-215.

37) Zweig, G. (1964) An SU(3) Model for Strong Interaction Symmetry and Its Breaking. CERN Report $8419 / \mathrm{TH} / 412$

38) Sugawara, H. (1968) A field theory of currents. Phys. Rev. 170, 1659-1662.

39) Hasert, F.J., Kabe, S., Krenz, W., Von Krogh, J., Lanske, D., Morfin, J. et al. (1973) Observation of neutrino-like interactions without muon or electron in the gargamelle neutrino experiment. Phys. Lett. 46B, 138-140; Hasert, F.J., Faissner, H., Krenz, W., Von Krogh, J., Lanske, D., Morfin, J. et al. (1973) Search for elastic muon-neutrino electron scattering. Phys. Lett. 46B, 121-124.

40) Kobayashi, M. and Maskawa, H. (1973) CP-Violation in the renormalizable theory of weak interaction. Prog. Theor. Phys. 49, 652-657.

41) Pakvasa, S. and Sugawara, H. (1976) $C P$ violation in the six-quark model. Phys. Rev. D 14, 305-308.

42) Pakvasa, S. (2009) The Kobayashi and Maskawa paper: the early days. Prog. Theor. Phys. 122, 31-36.

43) Pakvasa, S. and Sugawara, H. (1978) Discrete symmetry and Cabibbo angle. Phys. Lett. 73B, 61-64.

44) Sugawara, H. (2009) On KEK B-Factory. Prog. Theor. Phys. 122, 37-49.

45) Sugawara, H. (1980) QFD and unification. In Proceedings of the 20th High Energy Physics Conference, Madison Wisconsin, pp. 1095-1138.

46) Yoshimura, M. (1978) Unified gauge theories and the baryon number of the universe. Phys. Rev. Lett. 41, 281-284.

47) Watanabe, Y. (1979) In Proceedings of the Workshop on the Unified Theories and the Baryon Number in the Universe, Tsukuba, Japan (eds. Sawada, O. and Sugamoto, A.).

48) Banks, T., Fischler, W., Shenker, S. H. and Susskind, L. (1997) $M$ theory as a matrix model: A conjecture. Phys. Rev. D 55, 5112-5128.

49) Ishibashi, N., Kawai, H., Kitazawa, Y. and Tsuchiya, A. (1997) A large- $N$ reduced model as superstring. Nucl. Phys. B 498, 467-491.

50) Eguchi, T. and Kawai, H. (1982) Reduction of dynamical degrees of freedom in the large- $N$ gauge theory. Phys. Rev. Lett. 48, 1063-1066.

51) Sugawara, H. (2010) Matrix model and elliptic curve (arXiv:1001.0429v1 [hep-th]).

52) Sugawara, H. (2006) Quantum theory of DNA - An approach to electron transfer in DNA -. Prog. Theor. Phys. Suppl. 164, 17-27.

53) Sugawara, H. and Takahata, N. (to be published).

54) Sugawara, H. (2004 to 2008) Proceedings of the Workshop on "War and Peace", Sokendai, Hayama, Japan, series 2004, 2005, 2006, 2007, 2008, edited by Shimizu, Y.

(Received Dec. 28, 2009; accepted Feb. 6, 2010) 


\section{Profile}

Hirotaka Sugawara was born 1938 in Tokyo, Japan. He graduated from the University of Tokyo in 1961 and obtained Ph.D. from there in 1966. After postdoctoral positions at Cornell University, University of California at Berkeley, University of Chicago and Tokyo University of Education, he became Associate Professor at the Institute of Nuclear Study, University of Tokyo in 1971. He then became a professor at the Theoretical Physics Division of KEK (National Laboratory for High Energy Physics) in 1975. He served as the director of the KEK Physics Division from 1983 to 1989 and as director general of KEK from 1990 to 2003. After retirement from KEK, he was invited to become the Dai Ho Chun Visiting Professor at University of Hawaii (2003 to 2004). Then he served as an executive director of Sokendai (Graduate Univer-

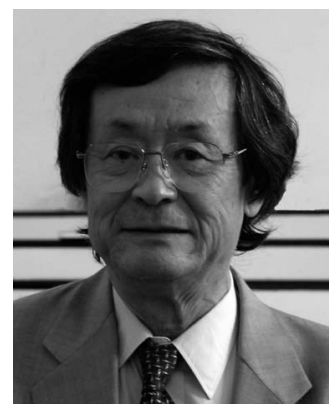
sity for Advanced Studies). Currently he is director of the JSPS Washington Office. His work in theoretical physics includes the following:

1. Collaborative work with Hironari Miyazawa on boson-fermion symmetry that can be characterized as a precursor to supersymmetry.

2. Discovery of the Sugawara construction that relates local currents to energy-momentum tensor.

3. Elaboration of the current algebra technique and its applications.

4. Elaboration of the physical consequences of the K-M model in collaboration with Sandip Pakvasa.

5. Application of discrete symmetry to the particle family problem.

He was given the Nishina Memorial Prize in 1971, the Toray Science and Technology Prize in 1996, and the Medal of Honor with Purple Ribbon in 1999. 